\title{
A GEOMETRIC APPROACH TO AN EQUATION OF J. D'ALEMBERT
}

\author{
A. PRÁSTARO AND TH. M. RASSIAS
}

(Communicated by Palle E. T. Jorgensen)

\begin{abstract}
By using a geometric framework of PDE's we prove that the set of solutions of the D'Alembert equation $(*)\left(\frac{\partial^{2} \log f}{\partial x \partial y}\right)=0$ is larger than the set of smooth functions of two variables $f(x, y)$ of the form $(* *) f(x, y)=$ $h(x) \cdot g(y)$. This agrees with a previous counterexample by Th. M. Rassias given to a statement by C. M. Stéphanos. More precisely, we have the following result.
\end{abstract}

Theorem. The set of 2-dimensional integral manifolds of PDE (*) properly contains the ones representable by graphs of 2-jet-derivatives of functions $f(x, y)$ expressed in the form $(* *)$.

A generalization of this result to functions of more than two variables is sketched also by considering the equation $\left(\frac{\partial^{n} \log f}{\partial x_{1} \cdots \partial x_{n}}\right)=0$.

\section{SYNOPSIS}

Questions of representation of functions in several variables by means of functions of a lesser number of variables have occupied the interest of mathematicians for centuries. One of these questions is closely connected with the thirteenth problem of Hilbert (1862-1943) and concerns the solvability of algebraic equations [7]. Functions of certain special forms have been investigated by several authors, including d'Alembert (1717-1783), who as early as 1747 proved that each sufficiently smooth scalar function $h$ of the form

$$
f(x, y)=h(x) \cdot g(y)
$$

has to satisfy the partial differential equation

$$
\left(\frac{\partial^{2} \log f}{\partial x \partial y}\right)=0
$$

(see [3]). This equation can be also expressed in the form

$$
\left|\begin{array}{ll}
f & f_{y} \\
f_{x} & f_{x y}
\end{array}\right|=0 .
$$

Received by the editors August 2, 1993.

1991 Mathematics Subject Classification. Primary 58G99; Secondary 58G18.

Key words and phrases. Geometry of partial differential equations, singular solutions of PDE's. 
A generalization of the form (1.1) to a finite sum of products of functions in single variables

$$
f(x, y)=\sum_{1 \leq i \leq n} h_{i}(x) \cdot g_{i}(y)
$$

has been considered since the beginning of this century, and it forms the fundamental problem in the subject. The functions of the tensor product (1.3) play a significant role in many areas of both pure and applied mathematics, for example, in the theory of integral equations, ordinary and partial equations, and the approximation theory. In the year 1904 in the section Arithmetics and Algebra at the 3rd International Congress of Mathematicians in Heidelberg, C. Stéphanos announced the following result:

The functions of the type (1.1) form the space of all solutions of the partial differential equation with the "Wronskian" of order $(k+1)$

$$
\operatorname{det} W_{n+1}(f)=\left|\begin{array}{cccc}
f & f_{y} & \cdots & f_{y^{n}} \\
f_{x} & f_{x y} & \cdots & f_{x y^{n}} \\
\cdots & & \cdots & \\
f_{x^{n}} & f_{x^{n} y} & \cdots & f_{x^{n} y^{n}}
\end{array}\right|=0 .
$$

His talk was published in [21] (see also [22]), where some further applications and consequences were discussed. However, no proof of the above result was given and no smoothness condition on the given function $h$ was mentioned. It is our belief that Stéphanos was thinking of analytic functions only. In fact, eighty years later, Rassias gave in [15] a counterexample of a function in two real variables satisfying the differential equation (1.2), which is not of the form (1.1). For the development of the subject see [13]. The new interest in the fundamental problem was revived in the beginning of the $1980 \mathrm{~s}$, when Neumann proved the basic theorem involving the equation (1.4) for functions of class $C^{n}$. This important result was published in [10] (see also [11]), where also a general criterion for a function $f: X \times Y \rightarrow \mathbb{R}$ (or $\mathbb{C}$, respectively), being of the form (1.3), was given in the form of the functional equation

$$
\left|\begin{array}{cccc}
f\left(x_{1}, y_{1}\right) & f\left(x_{1}, y_{2}\right) & \ldots & f\left(x_{1}, y_{n+1}\right) \\
f\left(x_{2}, y_{1}\right) & f\left(x_{2}, y_{2}\right) & \ldots & f\left(x_{2}, y_{n+1}\right) \\
\ldots & \ldots & \ldots & \ldots \\
f\left(x_{n+1}, y_{1}\right) & f\left(x_{n+1}, y_{2}\right) & \ldots & f\left(x_{n+1}, y_{n+1}\right)
\end{array}\right|=0
$$

for all $x_{t} \in X$ and $y_{j} \in Y$. For the proof of this criterion no structure on the sets $X$ and $Y$ is required. (Most of the theorems here hold for both realand complex-valued functions.) The problem of representing a function $f$ in several (more than two) variables by

$$
f\left(x_{1}, \ldots, x_{k}\right)=\sum_{1 \leq i \leq n} f_{i_{1}}\left(x_{1}\right) \cdots f_{i_{k}}\left(x_{k}\right)
$$

was proposed by Rassias in [16]. Gauchman and Rubel [4] obtained some new results on finite sums expansions of the form (1.3), especially for real analytic functions. Moreover, they developed an interesting approach to finding special solutions of the classical partial differential equations, the so-called extended separation of variables. The first existence theorem on the decomposition (1.5) 
was discovered by Neuman [12]. Later, Čadek and Šimša [1] found an effective criterion for the existence of the decomposition (1.5) by making use of a system of functional equations, which does not require any assumption on the function $f$. Furthermore, in [2] Čadek and Šimša outlined a way to find systems of partial differential equations whose solutions spaces form the family of all sufficiently smooth functions $f$ of type (1.5). Šimša [18] introduced some new functional expressions for functions of the form (1.3) using the so-called Casorati determinants. For a given function $f$ that cannot be expressed in the form (1.3) with a prescribed value of $f$, it is an interesting problem to find the best approximation of the type

$$
f(x, y)=\sum_{1 \leq i \leq n} f_{i}(x) \cdot f_{i}(y)
$$

in a given metric functional space. For the case of $L^{2}$ spaces, this problem was recently solved by Šmša [19]. However, for spaces without an inner product structure, the problem seems to be difficult and open. A weaker version of the above approximation problem, namely, to find a good approximation (1.6) and also an estimation of the error, was treated in [20] (see the forthcoming book by Rassias and Šimša [17]).

In this paper we prove the following theorem.

Theorem. The set Sol(d'A), of solutions of the d'Alembert equation, (d'A),

$$
\left(\frac{\partial^{2} \log f}{\partial x \partial y}\right)=0
$$

is larger than the set of functions which can be represented by (1.1).

This was made possible by using the modern geometric theory of PDE's [6, $8,9,14,24]$. In fact, we prove that (d'A) is a formally integrable PDE on the trivial fiber bundle $\pi: \mathbb{R}^{3} \rightarrow \mathbb{R}^{2}$. This means that for any point $q \in\left(\mathrm{d}^{\prime} \mathrm{A}\right)$ (initial condition), we can construct a formal solution, i.e., a tower of points $q_{k} \in\left(\mathrm{d}^{\prime} \mathrm{A}\right)_{+k} \equiv k$ th prolongation of $\left(\mathrm{d}^{\prime} \mathrm{A}\right)$, such that $\pi_{2+k, 2}\left(q_{k}\right)=q$, where $\pi_{2+k, 2}$ is the canonical projection

$$
\pi_{2+k, 2} ; J \mathscr{D}^{2+k}\left(\mathbb{R}^{2}, \mathbb{R}\right) \rightarrow J \mathscr{D}^{2}\left(\mathbb{R}^{2}, \mathbb{R}\right),
$$

where $J \mathscr{D}^{s}\left(\mathbb{R}^{2}, \mathbb{R}\right)$ denotes the $s$-order jet-derivative space for mappings $\mathbb{R}^{2}$ $\rightarrow \mathbb{R}$. In the analytic case this implies the complete integrability, i.e., the effective construction of analytic solutions in suitable neighbourhoods of any point $p \in \mathbb{R}^{2}$, with $p=\pi_{2}(q)$, where $\pi_{2}: J \mathscr{D}^{2}\left(\mathbb{R}^{2}, \mathbb{R}\right) \rightarrow \mathbb{R}^{2}$ is the canonical projection. Afterward, we prove that (d'A) can be identified with the set of points of $J \mathscr{D}^{2}\left(\mathbb{R}^{2}, \mathbb{R}\right)$ that can be represented in the form $\left(D^{2} f\right)(x, y)=$ $D^{2}(h \cdot g)(x, y)$, where $h=h(x)$ and $g=g(y)$. This, of course, implies that the set of functions of the form (1.1) are solutions of (d'A). On the other hand, any solution of (d'A) can be identified with 2-dimensional integral manifolds of the Cartan distribution $\mathbb{E}_{2} \subset T\left(\mathrm{~d}^{\prime} \mathrm{A}\right)$ of (d'A). Then, we show that such integral submanifolds cannot, in general, be represented as graphs of 2-jet derivatives of functions of the type (1.1). Therefore, the set of solutions of (d'A) is larger than the set of functions $f \in C^{\infty}\left(\mathbb{R}^{2}, \mathbb{R}\right)$ of the form (1.1). The above theorem can be generalized to functions of more than two variables. 


\section{Proof of THE THEOREM}

Lemma 1. Let $M_{i}, 1 \leq i \leq m$, be $C^{\infty}$ manifolds of finite dimension $n_{i}$. Then any $C^{\infty}$ function $f: M_{1} \times \cdots \times M_{m} \rightarrow \mathbb{R}$ can be represented in the following form:

$$
f=\sum_{1 \leq i \leq \infty} \alpha^{i_{1} \cdots i_{m}} f_{i_{1}} \cdots f_{i_{m}}, \quad \alpha^{i_{1} \cdots i_{m}} \in \mathbb{R}, \quad f_{i_{j}} \in C^{\infty}\left(M_{j}, \mathbb{R}\right), \quad 1 \leq j \leq m .
$$

Proof of Lemma 1. In fact one has the following canonical isomorphism of $\mathbb{R}$-vector spaces [23]

$$
\begin{aligned}
C^{\infty}\left(M_{1}, \mathbb{R}\right) \otimes_{\mathbf{R}} \cdots \otimes_{\mathbb{R}} C^{\infty}\left(M_{m}, \mathbb{R}\right) & \cong C^{\infty}\left(M_{1} \times \cdots \times M_{m}, \mathbb{R} \otimes_{\mathbb{R}} \cdots \otimes_{\mathbb{R}} \mathbb{R}\right) \\
& \cong C^{\infty}\left(M_{1} \times \cdots \times M_{m}, \mathbb{R}\right) .
\end{aligned}
$$

Thus any vector $f \in C^{\infty}\left(M_{1} \times \cdots \times M_{m}, \mathbb{R}\right)$ can be represented in the form

$$
f=\sum_{1 \leq i \leq \infty} \alpha^{i_{1} \cdots i_{m}} f_{i_{1}} \otimes \cdots \otimes f_{i_{m}} \cong \sum_{1 \leq i \leq \infty} \alpha^{i_{1} \cdots i_{m}} f_{i_{1}} \cdots f_{i_{m}},
$$

where $\left(f_{i_{j}}\right)$ is a $\mathbb{R}$-basis for $C^{\infty}\left(M_{1}, \mathbb{R}\right)$ and $\alpha^{i_{1} \cdots i_{m}} \in \mathbb{R}$.

Lemma 2. (1) Let $f_{i_{j}}, 1 \leq i_{j} \leq k_{j}<\infty$, be linear independent vectors of $C^{\infty}\left(M_{j}, \mathbb{R}\right), 1 \leq j \leq m$; then $f_{i_{1}} \cdots f_{i_{m}}$ generates a subvector space of $C^{\infty}\left(M_{1} \times \cdots \times M_{m}, \mathbb{R}\right)$ of dimension $k_{1} \cdots k_{m}$. Any vector

$$
f \in C^{\infty}\left(M_{1} \times \cdots \times M_{m}, \mathbb{R}\right)
$$

that belongs to such a subspace can be represented in the following way:

$$
\begin{aligned}
& f= \sum_{1 \leq i \leq k_{j}, 1 \leq j \leq m} \alpha^{i_{1} \cdots i_{m}} f_{i_{1}} \cdots f_{i_{m}}, \\
& \alpha^{i_{1} \cdots i_{m}} \in \mathbb{R}, \quad f_{i_{j}} \in C^{\infty}\left(\boldsymbol{M}_{j}, \mathbb{R}\right), \quad 1 \leq j \leq m .
\end{aligned}
$$

Thus a function can be represented in the form (2.1) iff $f$ belongs to some vector subspace of finite dimension $k_{1} \cdots k_{m}$.

(2) In particular, if $m=2, M_{1}=M_{2}=\mathbb{R}$, and $k_{1}, k_{2}=1$, then any one of such one-dimensional vector subspaces of $C^{\infty}(\mathbb{R}, \mathbb{R})$ is contained in $\operatorname{Sol}\left(\mathrm{d}^{\prime} \mathrm{A}\right)$.

Proof of Lemma 2. (1) It is a direct consequence of Lemma 1.

(2) In fact, the set of regular solutions of (d'A) contains all the functions $h(x, y)$ of the type $h(x, y)=\lambda f_{1}(x) f_{2}(y), \forall \lambda \in \mathbb{R}$.

Lemma 3. The d'Alembert equation (d'A) $\subseteq J \mathscr{D}^{2}\left(\mathbb{R}^{2}, \mathbb{R}\right)$ is a formally integrable, PDE of second order on the trivial fiber bundle $\pi: \mathbb{R}^{3} \rightarrow \mathbb{R}^{2}$.

Proof of Lemma 3. The symbol $\left(g_{2}\right)_{q}$ of (d'A) at $q \in\left(\mathrm{d}^{\prime} \mathrm{A}\right)$ is a vector subspace of $S_{2}^{0}\left(T_{p} M\right) \otimes v T_{u} W \cong \mathbb{R}^{2} \odot \mathbb{R}^{2}$, where $M \equiv \mathbb{R}^{2}, W \equiv \mathbb{R}^{3}, v T$ denotes the vertical tangent functor, $p=\pi_{2}(q)$, and $u=\pi_{2,0}(q)$; with respect to a coordinate system $\left\{x, y, u, u_{x}, u_{y}, u_{x x}, u_{x y}, u_{y y}\right\}$ on $J \mathscr{D}^{2}\left(\mathbb{R}^{2}, \mathbb{R}\right)$, we have that

$$
v=X^{x x} \partial u_{x x}+X^{x y} \partial u_{x y}+X^{y y} \partial u_{y y} \in\left(g_{2}\right)_{q}
$$

iff $v \cdot F=0$, where $F \equiv u u_{x y}-u_{x} u_{y}$. Thus $v \in\left(g_{2}\right)_{q}$ iff $X^{x y} u=0 \Leftrightarrow X^{x y}=0$. Hence

$$
\operatorname{dim}\left(g_{2}\right)_{q}=\operatorname{dim} \mathbb{R}^{2} \odot \mathbb{R}^{2}-1=3-1=2 .
$$


Furthermore,

$$
\operatorname{dim}\left(\mathrm{d}^{\prime} \mathrm{A}\right)=\operatorname{dim} J \mathscr{D}^{3}\left(\mathbb{R}^{2}, \mathbb{R}\right)-1=8-1=7 .
$$

Let us now consider the first prolongation $\left(d^{\prime} A\right)_{+1}$ of $\left(d^{\prime} A\right)$ :

$$
\left(\mathrm{d}^{\prime} \mathrm{A}\right)_{+1} \subset J \mathscr{D}^{3}\left(\mathbb{R}^{2}, \mathbb{R}\right): \mid \begin{gathered}
F \equiv u u_{x y}-u_{x} u_{y}=0, \\
F_{x} \equiv u u_{x y x}-u_{x x} u_{y}=0, \\
F_{y} \equiv u u_{x y y}-u_{x} u_{y y}=0 .
\end{gathered}
$$

Then we get $\operatorname{dim}\left(\mathrm{d}^{\prime} \mathrm{A}\right)_{+1}=\operatorname{dim} J \mathscr{D}^{3}\left(\mathbb{R}^{2}, \mathbb{R}\right)-3=12-3=9$. Moreover,

$$
v=X^{x x x} \partial u_{x x x}+X^{x x y} \partial u_{x x y}+X^{y y x} \partial u_{y y x}+X^{y y y} \partial u_{y y y} \in\left(\left(g_{2}\right)_{+1}\right)_{q}
$$

iff

$$
\begin{aligned}
\left\{v \cdot F=0, v \cdot F_{x}=0, v \cdot F_{y}=0\right\} & \Leftrightarrow\left\{u X^{x x y}=0, u X^{y y x}=0\right\} \\
& \Rightarrow \operatorname{dim}\left(\left(g_{2}\right)_{+1}\right)_{q}=4-2=2 .
\end{aligned}
$$

Therefore, we get

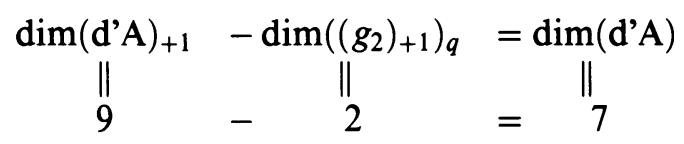

On the other hand we have the following exact sequence:

$$
\left(\text { d'A } _ { + 1 } \longrightarrow \left(\text { d'A }^{\stackrel{-k \rightarrow}{\longrightarrow}} F_{1}\right.\right.
$$

where:

(1) $F_{1}$ is a vector bundle on (d'A) canonically identified by means of the following commutative and exact diagrams of vector bundles over (d'A):

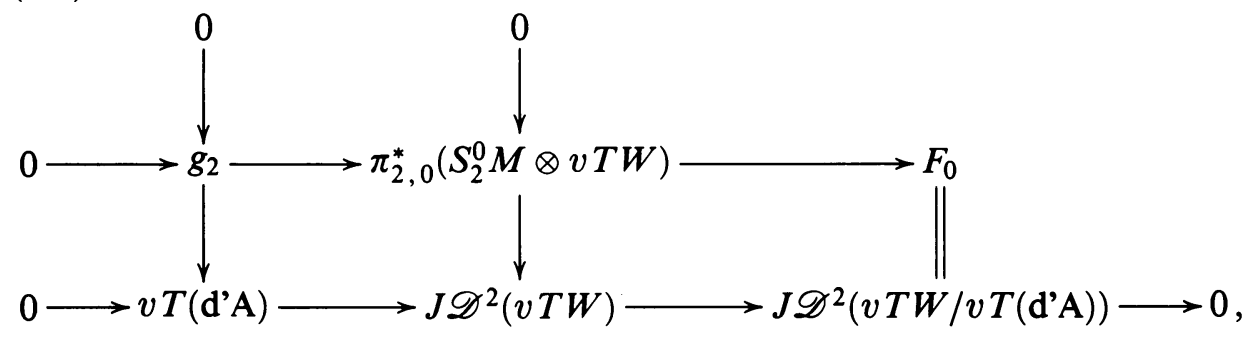

$$
0 \rightarrow\left(g_{2}\right)_{+1} \rightarrow \pi_{2,0}^{*}\left(S_{3}^{0} M \otimes v T W\right) \rightarrow T^{*} M \otimes F_{0} \rightarrow F_{1} ;
$$

and

(2) $k$ is the curvature of (d'A) [6]. Then we have

$$
\operatorname{dim} F_{1}=2 \cdot \operatorname{dim} F_{0}-\frac{(2+2) !}{3 !}+\operatorname{dim}\left(\left(g_{1}\right)_{+1}\right)_{q},
$$

where $\operatorname{dim} F_{0}=\operatorname{dim} J \mathscr{D}^{2}\left(\mathbb{R}^{2}, \mathbb{R}\right)-\operatorname{dim}\left(\mathrm{d}^{\prime} \mathrm{A}\right)=8-7=1$. Thus taking into account (2.2) we can conclude that $\operatorname{dim} F_{1}=0$, i.e., the curvature $k$ is zero; hence, the canonical mapping $\left(\mathrm{d}^{\prime} \mathrm{A}\right)_{+1} \rightarrow\left(\mathrm{d}^{\prime} \mathrm{A}\right)$ is surjective. Let us now consider the following fundamental relation [5]:

$$
\operatorname{dim}\left(\left(g_{2}\right)_{+1}\right)_{q} \leq \sum_{0 \leq i \leq 1} \operatorname{dim}\left(g_{2}\right)_{q}^{(i)},
$$


where $\left(g_{2}\right)_{q}^{(0)}=\left(g_{2}\right)_{q},\left(g_{2}\right)_{q}^{(i)} \equiv\left\{v \in\left(g_{2}\right)_{q} \mid\left\langle v, e_{1}\right\rangle=\cdots=\left\langle v, e_{i}\right\rangle=0\right\}, i>0$, being $\left\{e_{i}\right\}_{1 \leq i \leq 2}$ the basis on $T_{p} M$ induced by means of a coordinate system. If the equality holds, then the symbol $g_{2}$ is involutive. In fact, we get

$$
\begin{aligned}
& v=X^{x x} \partial u_{x x}+X^{y y} \partial u_{y y} \in\left(g_{2}\right)_{q}^{(1)} \quad \text { iff } \quad X^{x x}=0 \Rightarrow \operatorname{dim}\left(g_{2}\right)_{q}^{(1)}=1
\end{aligned}
$$

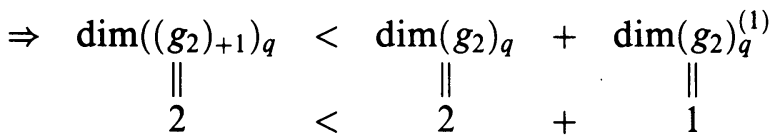

Thus (d'A) is not involutive. On the other hand we know [6] that we can prolong an equation a finite number of times such that it becomes involutive. Then, if $\left(\mathrm{d}^{\prime} \mathrm{A}\right)_{+r}$ has the symbol $\left(g_{2}\right)_{+r}$ involutive, we can find another integer $s$ such that $\left(\mathrm{d}^{\prime} \mathrm{A}\right){ }_{+r+s}^{(s)} \equiv \pi_{2+r+s, 2+r}\left(\left(\mathrm{~d}^{\prime} \mathrm{A}\right)_{+r+s}\right)$ is involutive formally integrable with the same regular solutions as (d'A). Therefore, we can conclude that (d'A) is formally integrable.

Lemma 4. (1) There exist two canonical embeddings of $J \mathscr{D}^{2}(\mathbb{R}, \mathbb{R})$ into $J \mathscr{D}^{2}\left(\mathbb{R}^{2}, \mathbb{R}\right)$ defined by

(a)

$$
a: J \mathscr{D}^{2}(\mathbb{R}, \mathbb{R}) \rightarrow J \mathscr{D}^{2}\left(\mathbb{R}^{2}, \mathbb{R}\right),\left(\xi, \zeta, \zeta_{\xi}, \zeta_{\xi \xi}\right) \mapsto\left(\xi, 0, \zeta, \zeta_{\xi}, 0, \zeta_{\xi \xi}, 0,0\right)
$$

and

(b)

$$
b: J \mathscr{D}^{2}(\mathbb{R}, \mathbb{R}) \rightarrow J \mathscr{D}^{2}\left(\mathbb{R}^{2}, \mathbb{R}\right),\left(\xi, \zeta, \zeta_{\xi}, \zeta_{\xi \xi}\right) \mapsto\left(0, \xi, \zeta, 0, \zeta_{\xi}, 0,0, \zeta_{\xi \xi}\right) .
$$

We call (a) the x-embedding and (b) the y-embedding respectively. Set $A^{2} \equiv$ $a\left(J \mathscr{D}^{2}(\mathbb{R}, \mathbb{R})\right), B^{2} \equiv b\left(J \mathscr{D}^{2}(\mathbb{R}, \mathbb{R})\right)$. Then we can identify $J \mathscr{D}^{2}(\mathbb{R}, \mathbb{R})$ with the two submanifolds $A^{2}$ and $B^{2}$ of $J \mathscr{D}^{2}\left(\mathbb{R}^{2}, \mathbb{R}\right)$, characterized by the following equations:

$$
\begin{gathered}
\left(\mathrm{A}^{2}\right):\left\{x-\xi=0, y=0, u-\zeta=0, u_{x}-\zeta_{\xi}=0, u_{y}=0,\right. \\
\left.u_{x x}-\zeta_{\xi \xi}=0, u_{x y}=0, u_{y y}=0\right\}, \\
\left(\mathrm{B}^{2}\right):\left\{x=0, y-\xi=0, u-\zeta=0, u_{x}=0, u_{y}-\zeta-\xi=0,\right. \\
\left.u_{x x}=0, u_{y y}-\zeta_{\xi \xi}=0\right\} .
\end{gathered}
$$

(2) One has $A^{2} \cap B^{2} \cong \mathbb{R}^{2}$, where $\mathbb{R}^{2}$ is the submanifold of $J \mathscr{D}^{2}\left(\mathbb{R}^{2}, \mathbb{R}\right)$ characterized by the equations $\left\{x=y=u_{x}=u_{y}=u_{x x}=u_{x y}=u_{y y}=0\right\}$.

Lemma 5. $A^{2}$ and $B^{2}$ are properly contained in (d'A): $A^{2} \subset\left(\mathrm{d}^{\prime} \mathrm{A}\right), B^{2} \subset$ (d'A).

Proof of Lemma 5. In fact, $\left(A^{2}\right)$ and $\left(B^{2}\right)$ satisfy the equation $u u_{x y}-u_{x} u_{y}=$ 0 .

Lemma 6. (1) There exist two canonical monomorphisms of $C^{\infty}(\mathbb{R}, \mathbb{R})$ into $C^{\infty}\left(\mathbb{R}^{2}, \mathbb{R}\right)$ defined by

$$
\begin{array}{ll}
\bar{a}: C^{\infty}(\mathbb{R}, \mathbb{R}) \rightarrow C^{\infty}\left(\mathbb{R}^{2}, \mathbb{R}\right), & \bar{a}(h)(x, y)=h(x), \\
\bar{b}: C^{\infty}(\mathbb{R}, \mathbb{R}) \rightarrow C^{\infty}\left(\mathbb{R}^{2}, \mathbb{R}\right), & \bar{b}(g)(x, y)=g(y) .
\end{array}
$$

We call $(\overline{\mathrm{a}})$ the $x$-embedding and $(\overline{\mathrm{b}})$ the y-embedding respectively. Set $A \equiv$ $\bar{a}\left(C^{\infty}(\mathbb{R}, \mathbb{R})\right), B \equiv \bar{b}\left(C^{\infty}(\mathbb{R}, \mathbb{R})\right)$. Then $C^{\infty}(\mathbb{R}, \mathbb{R})$ can be identified with two 
subvector spaces of $C^{\infty}\left(\mathbb{R}^{2}, \mathbb{R}\right): A$ is the subspace of functions $f(x, y)$ that are constant with respect to $y$-variable, and $B$ is the subspace of functions $f(x, y)$ that are constants with respect to the $x$-variable. One has $A \cap B=\mathbb{R}$, where $\mathbb{R}$ is identified with the subspace of $C^{\infty}\left(\mathbb{R}^{2}, \mathbb{R}\right)$ of constant functions.

(2) $A$ is the set of solutions of $A^{2}$, and $B$ is the set of solutions of $B^{2} . \mathbb{R}$ is the set of solutions of $A^{2} \cap B^{2}=\mathbb{R}$.

Lemma 7. (d'A) is diffeomorphic to the image $P^{2}$ of the canonical mapping

$$
j: J \mathscr{D}^{2}(\mathbb{R}, \mathbb{R}) \times J \mathscr{D}^{2}(\mathbb{R}, \mathbb{R}) \rightarrow J \mathscr{D}^{2}\left(\mathbb{R}^{2}, \mathbb{R}\right),
$$

defined by

$$
j\left(D^{2}\left(h(x), D^{2} g(y)\right)=D^{2}(h \cdot g)(x, y) .\right.
$$

More precisely the diagram

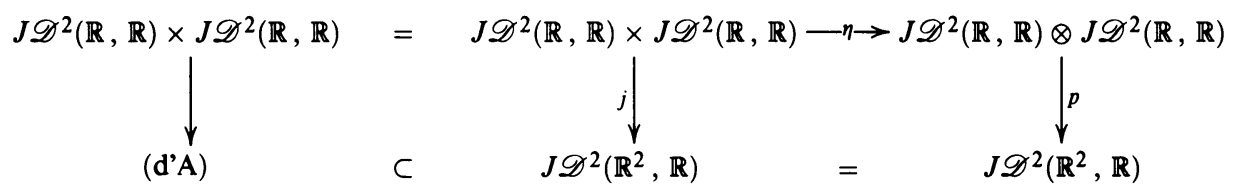

is commutative, where (i) $\eta$ is the canonical morphism of vector bundles over $\mathbb{R}^{2}(x, y)$, and (ii) $\rho$ is defined by the following equations:

$$
\begin{aligned}
& x \circ \rho=x, \quad y \circ \rho=y, \quad u \circ \rho=\bar{u} \overline{\bar{u}}, \quad u_{x} \circ \rho=\bar{u} \overline{\bar{u}}, \quad u_{x} \circ \rho=\bar{u}_{x} \overline{\bar{u}}, \\
& u_{y} \circ \rho=\bar{u} \overline{\bar{u}}_{y}, \quad u_{x x} \circ \rho=\bar{u}_{x x} \overline{\bar{u}}, \quad u_{y y} \circ \rho=\bar{u} \overline{\bar{u}}_{y y}, \quad u_{x y} \circ \rho=2 \bar{u} \overline{\bar{u}}_{y},
\end{aligned}
$$

where $\left(x, \bar{u}, \bar{u}_{x}, \bar{u}_{x x}, y, \overline{\bar{u}}, \overline{\bar{u}}_{y}, \overline{\bar{u}}_{y y}\right)$ are coordinates on $J \mathscr{D}^{2}(\mathbb{R}, \mathbb{R}) \times$ $J \mathscr{D}^{2}(\mathbb{R}, \mathbb{R})$.

Proof of Lemma 7. The image of $P^{2}$ of $\rho$ is the set of points $D^{2} f(x, y) \in$ $J \mathscr{D}^{2}\left(\mathbb{R}^{2}, \mathbb{R}\right)$ that can be written in the following way:

$$
D^{2} f(x, y)=D^{2}(h g)(x, y)
$$

where $h=h(x)$ and $g=g(y)$ are $C^{\infty}$-functions. Furthermore, $P^{2}$ is a seven-dimensional submanifold of $J \mathscr{D}^{2}\left(\mathbb{R}^{2}, \mathbb{R}\right)$ as $\rho$ has constant rank seven. Moreover, $P^{2} \subseteq\left(\mathrm{d}^{\prime} \mathrm{A}\right)$ as all points of $J \mathscr{D}^{2}\left(\mathbb{R}^{2}, \mathbb{R}\right)$ that satisfy (2.7) belong to (d'A) also. On the other hand the mapping $j$ is onto (d'A). In fact, for any point $q \in\left(\mathrm{d}^{\prime} \mathrm{A}\right)$ we can find a point $u \equiv\left(\left(D^{2} h\right)(x),\left(D^{2} g\right)(y)\right) \in$ $J \mathscr{D}^{2}(\mathbb{R}, \mathbb{R}) \times J \mathscr{D}^{2}(\mathbb{R}, \mathbb{R})$ such that $j(u)=q$. Indeed the point $u$ is given by

$$
u=\left(D^{2} f_{y}(x), D^{2} f_{x}(y)\right),
$$

where $f_{x}$ and $f_{y}$ are the partial mappings $\mathbb{R} \rightarrow \mathbb{R}$ at the points $x$ and $y$, respectively.

Lemma 8. The image of $C^{\infty}(\mathbb{R}, \mathbb{R}) \times C^{\infty}(\mathbb{R}, \mathbb{R})$ into $C^{\infty}\left(\mathbb{R}^{2}, \mathbb{R}\right)$ by means of the canonical mapping

$$
\begin{gathered}
c: C^{\infty}(\mathbb{R}, \mathbb{R}) \times C^{\infty}(\mathbb{R}, \mathbb{R}) \rightarrow C^{\infty}(\mathbb{R}, \mathbb{R}) \otimes_{\mathbf{R}} C^{\infty}(\mathbb{R}, \mathbb{R}) \\
\cong C^{\infty}\left(\mathbb{R}^{2}, \mathbb{R} \otimes_{\mathbb{R}} \mathbb{R}\right) \cong C^{\infty}\left(\mathbb{R}^{2}, \mathbb{R}\right)
\end{gathered}
$$

represents the set of solutions of (d'A) that can be represented in the form (1.1). Set $C \equiv c\left(C^{\infty}(\mathbb{R}, \mathbb{R}) \times C^{\infty}(\mathbb{R}, \mathbb{R})\right) \subset \operatorname{Sol}\left(\mathbf{d}^{\prime} A\right)$. 
Proof of Lemma 8. In fact, $f \in C^{\infty}\left(\mathbb{R}^{2}, \mathbb{R}\right)$ can be expressed in the form (1.1) iff $f \in C$. On the other hand from Lemma 7 it follows that if $f \in C$, the graph of $D^{2} f$ is contained in (d'A) $\subset J \mathscr{D}^{2}\left(\mathbb{R}^{2}, \mathbb{R}\right)$. Therefore, $C$ can be identified with the subset of Sol(d'A) defined by those integral manifolds of (d'A) that can be obtained as graphs of 2-jet-derivatives of functions $f(x, y)$ expressed in the form (1.1).

Lemma 9. Let $E_{2} \subset J \mathscr{D}^{2}(M, \mathbb{R})$ be a second-order $P D E$, where $M$ is an $n$ dimensional manifold. Let $F\left(x,{ }^{\alpha}, y, y_{\alpha}, y_{\alpha \beta}\right)=0$ be the local expression of $E_{2}$. Then, the space of characteristic vectors at a point $q \in E_{2}$ is an $n$ dimensional space generated by the following linearly independent vector fields on $E_{2}$ :

$$
\begin{aligned}
v^{\beta}= & \left(\partial y^{\alpha \beta} \cdot F\right) \partial x_{\alpha}+y_{\alpha}\left(\partial y^{\alpha \beta} \cdot F\right) \partial y+y_{\delta \alpha}\left(\partial y^{\delta \beta} \cdot F\right) \partial y^{\alpha} \\
& -\left[\left(\partial x_{\alpha} \cdot F\right)+y_{\alpha}(\partial y \cdot F)+y_{\omega \alpha}\left(\partial y^{\omega} \cdot F\right)\right] \partial y^{\alpha \beta} .
\end{aligned}
$$

Proof of Lemma 9. The ideal $\mathscr{J}\left(\mathbb{E}_{2}\right)$ that characterizes the Cartan distribution $\mathbb{E}_{2}$ of $E_{2}$ is generated by the following differential 1-forms:

$$
\begin{gathered}
\omega_{0} \equiv d F=\left(\partial x_{\alpha} \cdot F\right) d x^{\alpha}+(\partial y \cdot F) d y+\left(\partial y^{\alpha} \cdot F\right) d y_{\alpha}+\left(\partial y^{\alpha \beta} \cdot F\right) d y_{\alpha \beta} \\
\cdot \quad \omega_{1} \equiv d y-y_{\alpha} d x^{\alpha} ; \quad \omega_{2 \alpha} \equiv d y_{\alpha}-y_{\alpha \beta} d x^{\beta} \\
X=X^{\alpha} \partial x_{\alpha}+Y \partial y+Y_{\alpha} \partial y^{\alpha}+Y_{\beta \alpha} \partial y^{\alpha \beta} \in C^{\infty}\left(\mathbb{E}_{2}\right) \\
\left.\left.\Leftrightarrow X\rfloor \omega_{0}=X\right\rfloor \omega_{1}=X\right\rfloor \omega_{2 \alpha}=0 \\
\Rightarrow\left(\partial x_{\alpha} \cdot F\right) X^{\alpha}+(\partial y \cdot F) Y+\left(\partial y^{\alpha} \cdot F\right) Y_{\alpha}+\left(\partial y^{\alpha \beta} \cdot F\right) Y_{\alpha \beta}=0 \\
Y=y_{\alpha} X^{\alpha} ; \quad Y_{\alpha}=y_{\alpha \beta} X^{\beta}
\end{gathered}
$$

$X \in \operatorname{Char}\left(\mathbb{E}_{2}\right) \equiv$ set of characteristic vector fields of $\left.\mathbb{E}_{2} \Leftrightarrow X\right\rfloor d \mathscr{J}(\mathbb{E}) \subseteq \mathscr{J}\left(\mathbb{E}_{2}\right)$. Taking into account that $d \mathscr{J}\left(\mathbb{E}_{2}\right)$ is generated by the following differential forms:

$$
\begin{gathered}
\omega_{0}, \omega_{1}, \omega_{2 \alpha}, \omega_{3} \equiv d \omega_{1}=-d y_{\alpha} \wedge d x^{\alpha}, \omega_{4 \alpha} \equiv d \omega_{2 \alpha}=-d y_{\alpha \beta} \wedge d x^{\beta} \\
\Rightarrow \\
\left.\Rightarrow] \omega_{3}=A \omega_{0}+B \omega_{1}+C^{\alpha} \omega_{2 \alpha} ; X\right] \omega_{4 \alpha}=\bar{A}_{\alpha} \omega_{0}+\bar{B}_{\alpha} \omega_{1}+\bar{C}_{\alpha}^{\beta} \omega_{2 \beta} \\
\Rightarrow d x^{\alpha}\left[-Y_{\alpha}-A\left(\partial x_{\alpha} \cdot F\right)+B y_{\alpha}+C^{\delta} y_{\delta \alpha}\right]+d y[A(\partial y \cdot F)+B] \\
\quad+d y_{\alpha}\left[X^{\alpha}-A\left(\partial y^{\alpha} \cdot F\right)-C^{\alpha}\right]+d y_{\alpha \beta} A\left(\partial y^{\alpha \beta} \cdot F\right)=0 \\
\Rightarrow A=0 \Rightarrow C^{\alpha}=X^{\alpha} \Rightarrow B=0 \Rightarrow Y_{\alpha}=C^{\delta} y_{\delta \alpha} \Rightarrow Y_{\alpha}=Y^{\delta} y_{\delta \alpha} . \\
d x^{\beta}\left[-Y_{\alpha \beta}-\bar{A}_{\alpha}\left(\partial x_{\beta} \cdot F\right)+\bar{B}_{\alpha} y_{\beta}+\bar{C}_{\alpha}^{\omega} y_{\omega \beta}\right]+d y\left[\bar{A}_{\alpha}(\partial y \cdot F)+\bar{B}_{\alpha}\right] \\
\quad+d y_{\beta}\left[\bar{A}_{\alpha}\left(\partial y^{\beta} \cdot F\right)+\bar{C}_{\alpha}^{\beta}\right]+d y_{\omega \beta}\left[X^{\beta} \delta_{\alpha}^{\omega}-\bar{A}_{\alpha}\left(\partial y^{\omega \beta} \cdot F\right)\right]=0 \\
\Rightarrow \bar{B}_{\alpha}=-\bar{A}_{\alpha}(\partial y \cdot F) ; \bar{C}_{\alpha}^{\beta}=-\bar{A}_{\alpha}\left(\partial y^{\beta} \cdot F\right) ; X^{\beta} \delta_{\alpha}^{\omega}=\bar{A}_{\alpha}\left(\partial y^{\omega \beta} \cdot F\right) \\
\Rightarrow Y_{\alpha \beta}=-\bar{A}_{\alpha}\left[\left(\partial x_{\beta} \cdot F\right)+y_{\beta}(\partial y \cdot F)+y_{\omega \beta}\left(\partial y^{\omega} \cdot F\right)\right] \\
\Rightarrow X^{\alpha}=\bar{A}_{\beta}\left(\partial y^{\alpha \beta} \cdot F\right) ; Y=\bar{A}_{\beta} y_{\alpha}\left(\partial y^{\alpha \beta} \cdot F\right) ; Y_{\alpha}=\bar{A}_{\beta} y_{\delta \alpha}\left(\partial y^{\delta \beta} \cdot F\right) .
\end{gathered}
$$


Now we are able to prove that the set of solutions of (d'A) is larger than $C$. In fact, one can see that the Cartan distribution $\mathbb{E}_{2} \subset T$ (d'A) In (d'A), that characterizes the solutions of (d'A), is the annihilator of the ideal generated by the following differential 1-forms on $J \mathscr{D}^{2}\left(\mathbb{R}^{2}, \mathbb{R}\right)$ :

$$
\omega_{\alpha} \equiv \mid \begin{aligned}
& \omega_{0} \equiv d F=d u u_{x y}+u d u_{x y}-d u_{x} u_{y}-u_{x} d u_{y}, \\
& \omega_{1} \equiv d u-u_{x} d x-u_{y} d y \\
& \omega_{2} \equiv d u_{x}-u_{x x} d x-u_{x y} d y \\
& \omega_{3} \equiv d u_{y}-u_{y x} d x-u_{y y} d y
\end{aligned}
$$

Let us consider two 1-dimensional integral manifolds $V_{1}$ and $V_{2}$ of $A^{2}$ and $B^{2}$, respectively. Of course, one has $V_{1} \subset A^{2} \subset$ (d'A), $V_{2} \subset B^{2} \subset$ (d'A). Furthermore, $V_{i}, i=1,2$, are also 1-dimensional integral submanifolds of (d'A). Let $v_{i}, i=1,2$, be characteristic vector fields that are not contained in the tangent space $T V_{1}$. More precisely we can take

$$
\begin{aligned}
& v_{1} \equiv v^{x}=u\left[\partial y+u_{y} \partial u+u_{y x} \partial u_{x}+u_{y y} \partial u_{y}\right]+u_{x x} u_{y} \partial u_{x x}+u_{y y} u_{x} \partial u_{y x} \\
& v_{2} \equiv v^{y}=u\left[\partial x+u_{x} \partial u+u_{x x} \partial u_{x}+u_{x y} \partial u_{y}\right]+u_{x x} u_{y} \partial u_{x y}+u_{y y} u_{x} \partial u_{y y}
\end{aligned}
$$

Let $\phi_{i, t}:\left(\mathrm{d}^{\prime} \mathrm{A}\right) \rightarrow\left(\mathrm{d}^{\prime} \mathrm{A}\right)$ be the 1-parameter groups of diffeomorphisms generated by $v_{i}: \partial \phi_{i}=v_{i}$. Then $N_{i} \equiv \bigcup_{t} \phi_{i, t}\left(V_{i}\right), i=1,2$, are 2-dimensional integral manifolds of (d'A). Such manifolds cannot, in general, be represented as the graphs of the second-order jet-derivatives of functions $f(x, y)$ of the type (1.1).

Remark. This result interprets a general behaviour of PDE's. In fact, "singular solutions" or multivalued solutions cannot be interpreted as graphs of jet-derivative mappings. Therefore, a generalization of our considerations for functions of more than two variables can be, in a similar way, directly developed. In fact, we can also prove that the set of solutions of the following generalized D'Alembert equation $\left(\right.$ d'A $_{n} \subset J \mathscr{D}^{n}\left(\mathbb{R}^{n}, \mathbb{R}\right)$ :

$$
\left(\frac{\partial^{n} \log f}{\partial x_{1} \cdots \partial x_{n}}\right)=0
$$

properly contains graphs of the $n$-jet-derivatives of all functions $f \in$ $C^{\infty}\left(\mathbb{R}^{n}, \mathbb{R}\right)$ that can be expressed in the form $f\left(x^{1}, \ldots, x^{n}\right)=f_{1}\left(x^{1}\right) \cdots f_{n}\left(x^{n}\right)$. (We do not go into details as these are trivial extensions of previous ones made for two variables.)

The reader can find an extensive study, and other results, of this subject in the book by Th. M. Rassias and J. Šimša [17] (see in particular, Chapter VIII).

\section{REFERENCES}

1. M. Čadek and J. Šimša, Decomposable functions of several variables, Aequationes Math. 40 (1990), 8-25.

2. __ Decomposition of smooth functions of two multidimensional variables, Czechoslovak Math. J. 41 (1991), 342-358.

3. J. D'Alembert, Recherches sur la courbe que forme une corde tendue mise en vibration I-II, Hist. Acad. Berlin (1747), 214-249.

4. H. Gauchaman and L. A. Rubel, Sums of products of functions of $x$ times functions of $y$, Linear Algebra Appl. 125 (1989), 19-63. 
5. V. Guillemin and S. Sternberg, An algebraic model of transitive differential geometry, Bull. Amer. Math. Soc. 70 (1964), 16-28.

6. H. Goldschmidt, Integrability criteria for systems of nonlinear partial differential equations, J. Differential Geom. 1 (1967), 269-307.

7. D. Hilbert, Mathematical problems, Lecture delivered before the International Congress of Mathematicians at Paris in 1900, Bull. Amer. Math. Soc. 8 (1902), 437-479.

8. S. Krasil'shchik, V. Lychagin, and A. M. Vinogradov, Geometry of jet spaces and nonlinear partial differential equations, Gordon \& Breach, London, 1986.

9. V. Lychagin, Geometric theory of singularities of solutions of nonlinear differential equations, J. Soviet Math. 51 (1990), 2735-2757.

10. F. Neuman, Functions of two variables and matrices involving factorizations, C. R. Math. Rep. Acad. Sci. Canada 3 (1981), 7-11.

11. _ Factorizations of matrices and functions of two variables, Czechoslovak Math. J. 32 (1982), 582-588.

12. _ Finite sums of products of functions in single variables, Linear Algebra Appl. 134 (1990), 153-164.

13. F. Neuman and Th. M. Rassias, Functions decomposable into finite sums of products, Constantin Carathéodory: An International Tribute, vol. II, World Scientific, Singapore, 1991, pp. 956-963.

14. A. Prástaro, Quantum geometry of PDE's, Rep. Math. Phys. 30 (1991), 273-354.

15. Th. M. Rassias, A criterion for a function to be represented as a sum of products of factors, Bull. Inst. Math. Acad. Sinica 14 (1986), 377-382.

16. __ Problem P 286, Aequationes Math. 38 (1989), 111-112.

17. Th. M. Rassias and J. Šimša, Finite sums decompositions in mathematical analysis, Wiley, New York.

18. J. Šimša, A note on certain functional determinants, Aequationes Math. 44 (1992), 35-41.

19. quationes Math. 43 (1992), 248-263.

20. __ Stability of a differential criterion for decomposable functions of two variables (to appear).

21. C. M. Stéphanos, Sur une categorie d'équations fonctionelles, Rend. Circ. Mat. Palermo (2) 18 (1904), 360-362.

22. __ Sur une categorie d'equations fonctionnelles, Math. Kongress, Heidelberg (1904), 200-201; (1905)10, 194.

23. F. Treves, Topological vector spaces. Distributions and kernels, Academic Press, New York and London, 1967.

24. A. M. Vinogradov, Geometry of nonlinear differential equations, J. Soviet. Math. 17 (1981), 1624-1649.

Dipartimento di Metodi e Modelli Matematici per le Scienze Applicate, Universita' degli Studi di Roma "La Sapienza", Via A. Scarpa, 400161 Roma, Italy

Department of Mathematics, University of LaVerne, P. O. Box 51105, Kifissia, Athens, GREECE 14510 